\title{
Optimal Reinsurance-Investment Problem for an Insurer and a Reinsurer with Jump-Diffusion Process
}

\author{
Hanlei Hu ${ }^{(D}{ }^{1}$, Zheng Yin $(\mathbb{D})^{1}$ and Xiujuan Gao $\mathbb{D}^{2}$ \\ ${ }^{1}$ School of Economic Mathematics, Southwestern University of Finance and Economics, Chengdu 611130, China \\ ${ }^{2}$ School of Finance, Southwestern University of Finance and Economics, Chengdu 611130, China \\ Correspondence should be addressed to Hanlei Hu; huhanlei521@sina.com
}

Received 22 January 2018; Accepted 29 March 2018; Published 8 May 2018

Academic Editor: Francisco R. Villatoro

Copyright (c) 2018 Hanlei Hu et al. This is an open access article distributed under the Creative Commons Attribution License, which permits unrestricted use, distribution, and reproduction in any medium, provided the original work is properly cited.

\begin{abstract}
The optimal reinsurance-investment strategies considering the interests of both the insurer and reinsurer are investigated. The surplus process is assumed to follow a jump-diffusion process and the insurer is permitted to purchase proportional reinsurance from the reinsurer. Applying dynamic programming approach and dual theory, the corresponding Hamilton-Jacobi-Bellman equations are derived and the optimal strategies for exponential utility function are obtained. In addition, several sensitivity analyses and numerical illustrations in the case with exponential claiming distributions are presented to analyze the effects of parameters about the optimal strategies.
\end{abstract}

\section{Introduction}

The risk management is a significant issue for insurers. Reinsurance and investment are two effective ways to spread risks and gain profits. With reinsurance, the insurer shares part of the risks to the reinsurer and pays reinsurance premium as the compensation. Hence, the quest for optimal reinsurance-investment treaty becomes an attractive topic to both academics and practitioners. Several criteria have been selected to deal with the reinsurance and investment optimization problem. For example, the ruin probability and adjust coefficient are widely used for designing an optimal strategy. Schmidli [1] studies the proportional reinsurance and investment problem on minimizing the ruin probability. Promislow and Young [2] consider the optimal investment and quota-share reinsurance strategy for an insurer whose claim process is modeled by a Brownian motion with drift. Liang and Guo [3] discuss the ruin probability minimization and the adjustment coefficient maximization under the cases of diffusion approximation and jump-diffusion. The twodimensional compound Poisson risk model is considered in Li and Liu [4]. Luo and Taksar [5] obtain a closed form expression for the minimal absolute ruin function through solving an optimal control problem. The expected utility maximization is another tool to measure the objectives, which have attracted much attention. Browne [6] investigates the problem of maximizing the utility of terminal wealth and minimizing the probability of ruin for an insurance firm with a random risk process. A similar problem to that of Browne [6] for a jump-diffusion risk model is studied by Yang and Zhang [7]. Bai and Guo [8] consider an optimal investmentreinsurance problem with multiple risky assets and noshorting constraint. Cao and Wan [9] concern maximizing both exponential and power utilities of terminal wealth of an insurer with excepted value premium. Bauerle [10] obtains the optimal reinsurance strategy that minimizes the expected quadratic distance of the risk reserve under the CramerLundberg model (also see [11]). Chen and Yam [12] investigate an optimal investment-reinsurance problem with the meanvariance criterion in a regime-switching market. Zhao et al. [13] consider the default risk for mean-variance insurers for a jump-diffusion risk model. In addition, risk measures such as value-at-risk ( $\mathrm{VaR})$ and conditional value-at-risk (C-VaR) in quantifying financial and insurance risks are also used to analyze the optimal reinsurance problem (see Cai and Tan [14], Cai et al. [15], Balbs et al. [16], Tan et al. [17], and Chi and Tan [18]). 
The above-mentioned researches on optimal strategy are from the insurer's point of view. However, in practice, a reinsurance treaty involves two parties, an insurer and a reinsurer, which have conflicting interests. An optimal reinsurance treaty for an insurer may not be optimal for a reinsurer and it might be unacceptable for a reinsurer as pointed out by Borch [19]. Thus, it is necessary to consider the interest of the reinsurer. Works about optimal reinsurance-investment problem for both insurer and reinsurer have been developed in different ways. For instance, $\mathrm{Li}$ et al. [20] investigate the optimal investment problem for maximizing the product of the utility of an insurer and a reinsurer. Li et al. [21] discuss the time-consistent reinsurance-investment strategy for two parties under a mean-variance framework. Cai et al. [22] analyze the joint survival and profitable probabilities of the cedent and the reinsurer and establish sufficient conditions for the existence of optimal reinsurance treaties under a general reinsurance premium principle. Fang and $\mathrm{Qu}$ [23] derive the optimal reinsurance contract in the form of the combination of quota-share and stop-loss reinsurance with the optimization criteria that maximize the joint survival probability.

The aim of present work is to extend parts of works in Liang et al. [24] from two aspects. One is that we describe the insurer's wealth process by a jump-diffusion risk model, which contains a compound Poisson process perturbed by a Brownian motion. The other is that we investigate the optimal trading strategies taking the interests of both the insurer and reinsurer into account. Two wealth processes are formulated from the views of an insurer and a reinsurer, who are allowed to invest in the financial market. We study the optimal proportional reinsurance and investment problem for the jump-diffusion risk model, where the criterion of optimization is to maximize the excepted utility of the terminal wealth. The dynamic programming approach and dual theory are adopted to derive the optimal strategies for the insurer and reinsurer. Comparing and analyzing the retentions selected by the two parties, we find that there exists a game process between the insurer and the reinsurer.

The rest of this paper proceeds as follows. Section 2 describes the reinsurance-investment model with jumpdiffusion risk. Sections 3 and 4 derive the explicit expressions of the optimal reinsurance-investment strategies in the sense of maximizing the expected exponential utility of the terminal wealth for the insurer and the reinsurer, respectively. Section 5 provides several sensitivity analyses and numerical illustrations in the case with exponential claim distributions. Section 6 gives conclusions.

\section{The Reinsurance-Investment Model}

In this section, we formulate a continuous-time model where the insurer and reinsurer are allowed to trade in the insurance market and the financial market with no taxes or fees. Let $(\Omega, \mathscr{F}, P)$ be a complete probability space with filtration $\left\{\mathscr{F}_{t}, 0 \leq t \leq T\right\}$, which stands for the information available until time $t . T$ is a finite time horizon. All stochastic processes in this paper are supposed to be well defined in this probability space.

2.1. Wealth Process of the Insurer. Without reinsurance and investment, the surplus process of the insurer is described by the jump-diffusion model [7]

$$
d R(t)=c d t-d \sum_{i=1}^{N(t)} P_{i}+\beta d W_{1}(t)
$$

where $\sum_{i=1}^{N(t)} P_{i}$ is a compound Poisson process representing the cumulative amount of claims in time interval $[0, t]$, $\{N(t), t \geq 0\}$ is a homogeneous Poisson process with intensity $\lambda>0$, and $N(t)$ stands for the number of claims which arrive up to time $t$. The size of $i$ th claim $\left\{P_{i}, i \geq 1\right\}$ is a sequence of positive independent and identically distributed random variables with common distribution $F(p)$. Denote the mean value $E\left[P_{i}\right]=\mu$ and moment generating function $M_{P}(s)=E\left[e^{s P}\right] . \beta$ is a constant, $\left\{W_{1}(t), t \geq 0\right\}$ is a standard Brownian motion, and the diffusion term $\beta d W_{1}(t)$ represents the uncertainty related to the insurer's surplus process at time $t$. In addition, we assume that $\{N(t), t \geq 0\},\left\{P_{i}, i \geq 1\right\}$, and $\left\{W_{1}(t), t \geq 0\right\}$ are mutually independent. $c$ is premium rate of the insurer, which is calculated according to the expected value premium principle; that is, $c=(1+\theta) \lambda \mu$ with safety loading $\theta>0$.

The insurer is allowed to purchase proportional reinsurance from the reinsurer to transfer the underlying risk. For each $t \in[0, T]$, the risk exposure $q_{1}(t) \in[0,1]$ represents the retention level of reinsurance; that is, when the claim $P_{i}$ occurs, the insurer pays $q_{1}(t) P_{i}$ while the reinsurer pays $\left(1-q_{1}(t)\right) P_{i}$. Since the reinsurer shares the risk, the insurer should pay an additional cost in the form of reinsurance premium to the reinsurer. Suppose that the reinsurance premium principle is the same as that of the insurer, which is paid continuously at a constant rate $c_{1}=(1+\eta) \lambda \mu$ with safety loading $\eta>\theta$ of the reinsurer. The surplus process $R_{1}(t)$ associated with reinsurance strategy $q_{1}(t)$ of the insurer follows the dynamics

$$
\begin{aligned}
d R_{1}(t)= & c d t-q_{1}(t) d \sum_{i=1}^{N(t)} P_{i}+\beta d W_{1}(t) \\
& -\left(1-q_{1}(t)\right) c_{1} d t \\
= & {\left[\theta-\eta+q_{1}(t)(1+\eta)\right] \lambda \mu d t-q_{1}(t) d \sum_{i=1}^{N(t)} P_{i} } \\
& +\beta d W_{1}(t) .
\end{aligned}
$$

In addition to the reinsurance, the insurer is also allowed to invest in a financial market consisting of one risk-free asset and one risky asset. The price process of the risk-free asset $S_{0}(t)$ satisfies

$$
\begin{aligned}
d S_{0}(t) & =r S_{0}(t) d t \\
S_{0}(0) & =s_{0},
\end{aligned}
$$


where $r>0$ is the risk-free interest rate. The price process of the risky asset $S(t)$ is described by the geometric Brownian motion

$$
\begin{aligned}
d S(t) & =\alpha S(t) d t+\sigma S(t) d W(t), \\
S(0) & =s,
\end{aligned}
$$

where $\alpha>r$ and $\sigma$ are positive constants, $W(t)$ is another standard Brownian motion defined on $(\Omega, \mathscr{F}, P)$, which is related to $W_{1}(t)$ by $E\left[d W_{1}(t) W(t)\right]=\rho d t$, and $\rho \in[-1,1]$ is the correlation coefficient.

Let $\pi_{1}(t)$ be the amount of the wealth invested in the risky asset at time $t$ by the insurer. Then the remainder $X(t)-$ $\pi_{1}(t)$ is invested in the risk-free asset. Since the insurer is allowed to purchase reinsurance and invest in the financial market, the trading strategy is a pair of stochastic processes $\Pi_{1}:=\left(q_{1}(t), \pi_{1}(t)\right), t \in[0, T]$, where $q_{1}(t)$ represents the reinsurance strategy and $\pi_{1}(t)$ represents the investment strategy. The reinsurance-investment strategy $\Pi_{1}(t)$ is said to be admissible if it is $\mathscr{F}_{t}$-progressively measurable and satisfies $0 \leq q_{1}(t) \leq 1, E\left[\int_{0}^{T} \pi_{1}^{2}(t) d t\right]<\infty$. Corresponding to an admissible reinsurance-investment strategy $\Pi_{1}$ and the initial wealth $X(0)=x$, the wealth process $X(t)$ of the insurer follows the stochastic differential equation

$$
\begin{aligned}
& d X(t)=d R_{1}(t)+\pi_{1}(t) \frac{d S(t)}{S(t)}+(X(t) \\
& \left.-\pi_{1}(t)\right) \frac{d S_{0}(t)}{S_{0}(t)}=\left[r X(t)+(\alpha-r) \pi_{1}(t)\right. \\
& \left.\quad+(\theta-\eta) \lambda \mu+q_{1}(t)(1+\eta) \lambda \mu\right] d t \\
& -q_{1}(t) d \sum_{i=1}^{N(t)} P_{i}+\beta d W_{1}(t)+\sigma \pi_{1}(t) d W(t) .
\end{aligned}
$$

Suppose that the utility function $U_{1}(\cdot)$ is concave and continuously differentiable on $R$. The objective of the insurer is assumed to maximize the expected utility of terminal wealth $X(T)$; that is,

$$
\max _{q_{1}, \pi_{1}} E\left[U_{1}(X(T))\right],
$$

subject to (5).

2.2. Wealth Process of the Reinsurer. In the presence of the proportional reinsurance contract, the surplus process $R_{2}(t)$ of the reinsurer satisfies

$$
\begin{aligned}
d R_{2}(t)= & \left(1-q_{2}(t)\right)(1+\eta) \lambda \mu d t \\
& -\left(1-q_{2}(t)\right) d \sum_{i=1}^{N(t)} P_{i},
\end{aligned}
$$

where $q_{2}(t)$ is the reinsurance strategy chosen by the reinsurer. Generally, the reinsurer will accept the reinsurance proportion proposed by the insurer when the retention level of the reinsurer is smaller than that of the insurer, while in the opposite case, in order to prevent large losses, the reinsurer may not accept the reinsurance strategy given by the insurer.

Let $\pi_{2}(t)$ be the amount of the wealth invested in the risky asset at time $t$ by the reinsurer. Then the remainder $Y(t)-\pi_{1}(t)$ is invested in the risk-free asset. Similar to the insurer, the trading strategy of the reinsurer is also a pair of processes $\Pi_{2}:=\left(q_{2}(t), \pi_{2}(t)\right), t \in[0, T]$, where $q_{2}(t)$ and $\pi_{2}(t)$ represent the reinsurance strategy and the investment strategy, respectively. The reinsurance-investment strategy $\Pi_{2}(t)$ is said to be admissible if it is $\mathscr{F}_{t}$-progressively measurable and satisfies $0 \leq q_{2}(t) \leq 1, E\left[\int_{0}^{T} \pi_{2}^{2}(t) d t\right]<$ $\infty$. Corresponding to an admissible reinsurance-investment strategy $\Pi_{2}$ and the initial wealth $Y(0)=y$, the wealth process $Y(t)$ of the reinsurer follows the stochastic differential equation

$$
\begin{aligned}
& d Y(t) \\
& =\left[r Y(t)+(\alpha-r) \pi_{2}(t)+\lambda \mu\left(1-q_{2}(t)\right)(1+\eta)\right] d t \\
& \quad-\left(1-q_{2}(t)\right) d \sum_{i=1}^{N(t)} P_{i}+\sigma \pi_{2}(t) d W(t) .
\end{aligned}
$$

Suppose that the utility function $U_{2}(\cdot)$ is concave and continuously differentiable on $R$. The objective of the reinsurer is assumed to maximize the expected utility of terminal wealth $Y(T)$; that is,

$$
\max _{q_{2}, \pi_{2}} E\left[U_{2}(Y(T))\right]
$$

subject to (8).

\section{Optimal Strategy for the Insurer}

Assume that the insurer takes an exponential utility function

$$
U_{1}(x)=\lambda_{1}-\frac{m}{\gamma_{1}} e^{-\gamma_{1} x}, \quad \lambda_{1}, m>0
$$

where $\gamma_{1}>0$ represents the absolute risk aversion coefficient. The exponential utility plays a prominent role in insurance mathematics and actuarial practice. It is the only utility function under which the principle of "zero utility" gives a fair premium that is dependent on the level of reserve of an insurance company.

For an admissible strategy $\left(q_{1}, \pi_{1}\right) \in \Pi_{1}$, define the value function

$$
H(t, x)=\sup _{\left(q_{1}, \pi_{1}\right)} E\left[U_{1}(X(T)) \mid X(t)=x\right]
$$

$$
0 \leq t \leq T
$$

with $H(T, x)=U_{1}(x)$. According to works of Soner [25], if the value function $H(t, x)$ and its partial derivatives $H_{t}, H_{x}$, 
and $H_{x x}$ are continuous on $[0, T] \times R^{+}$, then $H(t, x)$ satisfies the following Hamilton-Jacobi-Bellman (HJB) equation

$$
\begin{aligned}
& H_{t}+[r x+(\theta-\eta) \lambda \mu] H_{x}+\frac{\beta^{2}}{2} H_{x x} \\
& +\sup _{\pi_{1}(t) \geq 0}\left\{(\alpha-r) \pi_{1}(t) H_{x}\right. \\
& \left.+\left(\frac{1}{2} \sigma^{2} \pi_{1}^{2}(t)+\rho \beta \sigma \pi_{1}(t)\right) H_{x x}\right\} \\
& +\sup _{q_{1}(t) \in[0,1]}\left\{q_{1}(t)(1+\eta) \lambda \mu H_{x}\right. \\
& \left.+\lambda E\left[H\left(t, x-q_{1}(t) P\right)-H(t, x)\right]\right\}=0
\end{aligned}
$$

with boundary condition $H(T, x)=U_{1}(x)$, for any $(t, x) \epsilon$ $[0, T) \times R^{+}$.

Differentiating (12) with respect to $\pi_{1}(t)$ and setting the derivatives equal to zero, we have

$$
(\alpha-r) H_{x}+\left(\sigma^{2} \pi_{1}(t)+\rho \beta \sigma\right) H_{x x}=0 .
$$

The first-order maximizing condition for the insurer's investment strategy $\pi_{1}(t)$ yields

$$
\pi^{*}(t)=-\frac{\rho \beta \sigma}{\sigma^{2}}-\frac{\alpha-r}{\sigma^{2}} \frac{H_{x}}{H_{x x}} .
$$

Substituting (14) into the left-hand side of the HJB equation (12), we obtain

$$
\begin{aligned}
H_{t} & +[r x+(\theta-\eta) \lambda \mu] H_{x}+\frac{\beta^{2}}{2} H_{x x} \\
& -\frac{\left[(\alpha-r) H_{x}+\rho \beta \sigma H_{x x}\right]^{2}}{2 \sigma^{2} H_{x x}} \\
& +\sup _{q_{1}(t) \in[0,1]}\left\{q_{1}(t)(1+\eta) \lambda \mu H_{x}\right. \\
& \left.+\lambda E\left[H\left(t, x-q_{1}(t) P\right)-H(t, x)\right]\right\}=0
\end{aligned}
$$

with $H(T, x)=U_{1}(x)$. We construct a solution for (15) in the following form:

$$
H(t, x)=\lambda_{1}-\frac{m}{\gamma_{1}} \exp \left[-\gamma_{1} x e^{r(T-t)}+h(T-t)\right],
$$

where $h(\cdot)$ is a suitable function such that expression (16) is a solution of (15). The boundary condition $H(T, x)=U_{1}(x)$ implies $h(T)=0$. From (16), we have

$$
\begin{aligned}
H_{t} & =\left(H(t, x)-\lambda_{1}\right)\left[\gamma_{1} x r e^{r(T-t)}-h^{\prime}(T-t)\right], \\
H_{x} & =\left(H(t, x)-\lambda_{1}\right)\left[-\gamma_{1} e^{r(T-t)}\right], \\
H_{x x} & =\left(H(t, x)-\lambda_{1}\right)\left[\gamma_{1}^{2} e^{2 r(T-t)}\right], \\
E & {\left[H\left(t, x-q_{1}(t) P\right)-H(t, x)\right] } \\
& =\left(H(t, x)-\lambda_{1}\right)\left[M_{P}\left(\gamma_{1} q_{1}(t) e^{r(T-t)}\right)-1\right] .
\end{aligned}
$$

Substituting (17) into (15) yields

$$
\begin{aligned}
H_{t}+ & {[r x+(\theta-\eta) \lambda \mu] H_{x}+\frac{\beta^{2}}{2} H_{x x} } \\
& -\frac{\left[(\alpha-r) H_{x}+\rho \beta \sigma H_{x x}\right]^{2}}{2 \sigma^{2} H_{x x}}+\sup _{q_{1}(t) \in[0,1]} f\left(q_{1}(t)\right) \\
= & 0,
\end{aligned}
$$

where

$$
\begin{aligned}
& f\left(q_{1}(t)\right)=\lambda\left(H(t, x)-\lambda_{1}\right) \\
& \quad \cdot\left[-\mu \gamma_{1}(1+\eta) q_{1}(t) e^{r(T-t)}+M_{P}\left(\gamma_{1} q_{1}(t) e^{r(T-t)}\right)\right. \\
& \quad-1] .
\end{aligned}
$$

Differentiating (19) with respect to $q_{1}(t)$, we get

$$
\begin{gathered}
\frac{\partial f\left(q_{1}(t)\right)}{\partial q_{1}(t)}=\lambda \gamma_{1} e^{r(T-t)}\left(H(t, x)-\lambda_{1}\right) \\
\cdot\left[-\mu(1+\eta)+E\left[P e^{\gamma_{1} q_{1}(t) P e^{r(T-t)}}\right]\right], \\
\frac{\partial^{2} f\left(q_{1}(t)\right)}{\partial q_{1}^{2}(t)}=\lambda \gamma_{1}^{2} e^{2 r(T-t)}\left(H(t, x)-\lambda_{1}\right) \\
\cdot E\left[P^{2} e^{\gamma_{1} q_{1}(t) P e^{r(T-t)}}\right]<0 .
\end{gathered}
$$

Thus, $f\left(q_{1}(t)\right)$ is concave and its maximizer $q_{1}(t)$ satisfies the equation

$$
(1+\eta) \mu=E\left[P e^{\gamma_{1} q_{1}(t) P e^{r(T-t)}}\right]=M_{P}^{\prime}(\epsilon),
$$

where $\epsilon:=\gamma_{1} q_{1}(t) e^{r(T-t)}$.

Lemma 1. Equation (21) has a unique positive root $\epsilon$.

Proof. Let

$$
v(\epsilon)=(1+\eta) \mu-M_{P}^{\prime}(\epsilon) .
$$

From

$$
\begin{gathered}
v^{\prime}(\epsilon)=-E\left[P^{2} e^{\epsilon P}\right]<0, \\
v^{\prime \prime}(\epsilon)=-E\left[P^{3} e^{\epsilon P}\right]<0,
\end{gathered}
$$

we know that $v(\epsilon)$ is a decreasing concave function. Moreover, $v(0)=\mu \eta>0$. Thus $v(\epsilon)$ has a unique point of intersection with the abscissa axis, which implies that (21) has a unique positive solution $\epsilon$.

Note that $q_{1}(t)=\left(\epsilon / \gamma_{1}\right) e^{-r(T-t)}$ and the optimal reinsurance proportion $q_{1}^{*}(t) \in[0,1]$. If $0 \leq q_{1}(t) \leq 1$, the optimal reinsurance proportion $q_{1}^{*}(t)$ coincides with $q_{1}(t)$. If $q_{1}(t) \leq 0$, we let $q_{1}^{*}(t)=0$. If $q_{1}(t) \geq 1$, we set $q_{1}^{*}(t)=1$. In this paper, $q_{1}(t)=0$ means that the reinsurer takes all 
the insurance risk and the insurer acts as a middleman in the process of the insurance. From Lemma $1, \epsilon$ is a positive constant which depends on the safety loading $\eta$ and the claim sizes distribution $F(p)$. Therefore, $q_{1}(t) \geq 0$ and we discuss the optimal reinsurance strategy for the insurer in the following three cases.

When $\epsilon \leq \gamma_{1}$, we have $\epsilon / \gamma_{1} \leq 1$ and $q_{1}(t)=$ $\left(\epsilon / \gamma_{1}\right) e^{-r(T-t)} \leq 1$ for any $t \in[0, T]$. Then the optimal reinsurance strategy is $q_{1}^{*}(t)=q_{1}(t), 0 \leq t \leq T$.

When $\gamma_{1} \leq \epsilon \leq \gamma_{1} e^{r T}$, we have $\epsilon / \gamma_{1}>1$. Let $t_{0}=T+$ $(1 / r) \ln \left(\gamma_{1} / \epsilon\right)$. Then $q_{1}(t)=\left(\epsilon / \gamma_{1}\right) e^{-r(T-t)}<1$ for $t \in\left[0, t_{0}\right]$

and $q_{1}(t) \geq 1$ for $t \in\left[t_{0}, T\right]$; the optimal reinsurance strategy is

$$
q_{1}^{*}(t)= \begin{cases}q_{1}(t), & 0 \leq t \leq t_{0} \\ 1, & t_{0} \leq t \leq T .\end{cases}
$$

When $\epsilon \geq \gamma_{1} e^{r T}$, we have $q_{1}(t)=\left(\epsilon / \gamma_{1}\right) e^{-r(T-t)} \geq e^{r t} \geq 1$ for any $t \in[0, T]$. Then the optimal reinsurance strategy is $q_{1}^{*}(t)=1,0 \leq t \leq T$. by

Substituting $q_{1}^{*}(t)$ into (19), the value of $f\left(q_{1}^{*}(t)\right)$ is given

$$
f\left(q_{1}^{*}(t)\right)= \begin{cases}\lambda\left(H(t, x)-\lambda_{1}\right)\left[-\epsilon \mu(1+\eta)+M_{P}(\epsilon)-1\right], & q_{1}^{*}(t)=q_{1}(t), \\ \lambda\left(H(t, x)-\lambda_{1}\right)\left[-\bar{\epsilon} \mu(1+\eta)+M_{P}(\bar{\epsilon})-1\right], & q_{1}^{*}(t)=1,\end{cases}
$$

where $\bar{\epsilon}:=\gamma_{1} e^{r(T-t)}$.

Replacing the supremum in (18) by $f\left(q_{1}^{*}(t)\right)$ yields the following equation:

$$
\begin{aligned}
H_{t}+ & {[r x+(\theta-\eta) \lambda \mu] H_{x}+\frac{\beta^{2}}{2} H_{x x} } \\
& -\frac{\left[(\alpha-r) H_{x}+\rho \beta \sigma H_{x x}\right]^{2}}{2 \sigma^{2} H_{x x}}+f\left(q_{1}^{*}(t)\right)=0 .
\end{aligned}
$$

Here, the stochastic optimal control problem described above has been transformed into solving a partial differential equation for the value function $H(t, x)$. We intent to find the solution to (26) with boundary condition $H(T, x)=U_{1}(x)$ by using Legendre transformation and variables change method.

Definition 2 (see [26]). Let $H: R \rightarrow R$ be a convex function. For $z>0$, define the Legendre transform

$$
L(z)=\sup _{x>0}\{H(x)-z x\}
$$

The function $L(z)$ is called the Legendre dual of function $H(x)$.

Following the works in [26], we define a Legendre transform

$$
\begin{aligned}
\widehat{H}(t, z) & =\sup _{x>0}\{H(t, x)-z x\}, \quad t \in[0, T], \\
g(t, z) & =\inf _{x>0}\{x \mid H(t, x) \geq z x+\widehat{H}(t, z)\},
\end{aligned}
$$

$$
t \in[0, T],
$$

where $z>0$ denotes the dual variable to $x$. The function $\widehat{H}(t, z)$ is related to $g(t, z)$ by

$$
g(t, z)=-\widehat{H}_{z}(t, z) .
$$

Noting that $H(T, x)=U_{1}(x)$ at terminal time $T$, we have

$$
\begin{aligned}
\widehat{H}(T, z) & =\sup _{x>0}\left\{U_{1}(x)-z x\right\}, \\
g(T, z) & =\inf _{x>0}\left\{x \mid U_{1}(x) \geq z x+\widehat{H}(T, z)\right\},
\end{aligned}
$$

from which we get

$$
g(T, z)=\left(U_{1}^{\prime}\right)^{-1}(z) .
$$

Equation (31) shows that $g(T, z)$ is the inverse of marginal utility.

Using (28), we derive $H_{x}(t, x)=z$, and

$$
\begin{aligned}
g(t, z) & =x, \\
\widehat{H}(t, z) & =H(t, g)-z g .
\end{aligned}
$$

Referring to [26], we get the following transformation rules:

$$
\begin{aligned}
H_{t} & =\widehat{H}_{t}, \\
H_{x x} & =-\frac{1}{\widehat{H}_{z z}},
\end{aligned}
$$

where $\widehat{H}=\widehat{H}(t, z)$. Substituting (33) into (26), we have

$$
\begin{aligned}
\widehat{H}_{t}+ & {[r g(t, z)+(\theta-\eta) \lambda \mu] z+\frac{\beta^{2}\left(1-\rho^{2}\right)}{2} H_{x x} } \\
& -\frac{(\alpha-r)^{2} z^{2} \widehat{H}_{z z}}{2 \sigma^{2}}-\frac{\rho \beta(\alpha-r) z}{\sigma} \\
& +\lambda\left(\widehat{H}(t, z)+z g(t, z)-\lambda_{1}\right) f_{1}\left(q_{1}^{*}(t)\right)=0,
\end{aligned}
$$

where

$$
\begin{aligned}
f_{1} & \left(q_{1}^{*}(t)\right) \\
& = \begin{cases}-\epsilon \mu(1+\eta)+M_{P}(\epsilon)-1, & q_{1}^{*}(t)=q_{1}(t), \\
-\bar{\epsilon} \mu(1+\eta)+M_{P}(\bar{\epsilon})-1, & q_{1}^{*}(t)=1 .\end{cases}
\end{aligned}
$$


Letting $\rho^{2}=1$ and differentiating (34) with respect to $z$ yields

$$
\begin{aligned}
g_{t}(t, z) & -r g(t, z) \\
+ & {\left[\frac{(\alpha-r)^{2}}{\sigma^{2}}-r-\lambda f_{1}\left(q_{1}^{*}(t)\right)\right] z g_{z}(t, z) } \\
+ & \frac{(\alpha-r)^{2} z^{2}}{2 \sigma^{2}} g_{z z}(t, z)+\frac{\rho \beta(\alpha-r)}{\sigma} \\
& -(\theta-\eta) \lambda \mu=0
\end{aligned}
$$

with boundary condition $g(T, z)=\left(U_{1}^{\prime}\right)^{-1}(z)$.

The optimal investment strategy (14) is in the form

$$
\pi^{*}(t)=-\frac{\rho \beta \sigma}{\sigma^{2}}-\frac{\alpha-r}{\sigma^{2}} z g_{z}(t, z) .
$$

The problem now is to solve (36) for the dual $g(t, z)$ and replace it in (37) so as to obtain the optimal strategy.

From the exponential utility function given by (10) and (31), the boundary condition is expressed as

$$
g(T, z)=-\frac{1}{\gamma_{1}} \ln \frac{z}{m} .
$$

Thus, we try to conjecture a solution to (36) in the form

$$
g(t, z)=-\frac{a(t)}{\gamma_{1}} \ln \frac{z}{m}+b(t)
$$

with $a(T)=1$ and $b(T)=0$. Then we have

$$
\begin{aligned}
g_{t}(t, z) & =-\frac{a^{\prime}(t)}{\gamma_{1}} \ln \frac{z}{m}+b^{\prime}(t), \\
g_{z}(t, z) & =-\frac{a(t)}{\gamma_{1} z} \\
g_{z z}(t, z) & =\frac{a(t)}{\gamma_{1} z^{2}} .
\end{aligned}
$$

Substituting (40) into (36) yields

$$
\begin{gathered}
-\frac{\ln (z / m)}{\gamma_{1}} a^{\prime}(t)+b^{\prime}(t)+\frac{r \ln (z / m)}{\gamma_{1}} a(t)-r b(t) \\
-\left[\frac{(\alpha-r)^{2}}{2 \sigma^{2}}-r-\lambda f_{1}\left(q_{1}^{*}(t)\right)\right] \frac{a(t)}{\gamma_{1}} \\
+\frac{\rho \beta(\alpha-r)}{\sigma}-(\theta-\eta) \lambda_{\mu}=0 .
\end{gathered}
$$

We split (41) into two equations

$$
\begin{aligned}
& a^{\prime}(t)-r a(t)=0, \\
& b^{\prime}(t)-r b(t)-\left[\frac{(\alpha-r)^{2}}{2 \sigma^{2}}-r-\lambda f_{1}\left(q_{1}^{*}(t)\right)\right] \frac{a(t)}{\gamma_{1}} \\
& \quad+\frac{\rho \beta(\alpha-r)}{\sigma}-(\theta-\eta) \lambda \mu=0 .
\end{aligned}
$$

Taking the boundary conditions $a(T)=1$ and $b(T)=0$ into account, the solutions to (42) are

$$
\begin{aligned}
& a(t)=e^{r(t-T)}, \\
& b(t)=\frac{A\left(1-e^{r(t-T)}\right)}{r}+\frac{B(t-T)}{\gamma_{1}} e^{r(t-T)},
\end{aligned}
$$

where $A=\rho \beta(\alpha-r) / \sigma-(\theta-\eta) \lambda \mu$ and $B=(\alpha-r)^{2} / 2 \sigma^{2}-$ $r-\lambda f_{1}\left(q_{1}^{*}(t)\right)$.

The above derivations lead to the following theorem.

Theorem 3. Assume that the insurer takes an exponential utility function in the form of (10). Let $t_{0}=T+(1 / r) \ln \left(\gamma_{1} / \epsilon\right)$ and $\rho^{2}=1$, the optimal reinsurance-investment strategies for the insurer are given as follows:

(1) If $\epsilon \leq \gamma_{1}$, the optimal reinsurance strategy is $q_{1}^{*}(t)=$ $\left(\epsilon / \gamma_{1}\right) e^{-r(T-t)}, \quad 0 \leq t \leq T$.

(2) If $\gamma_{1} \leq \epsilon \leq \gamma_{1} e^{r T}$, the optimal reinsurance strategy is

$$
q_{1}^{*}(t)= \begin{cases}\frac{\epsilon}{\gamma_{1}} e^{-r(T-t)}, & 0 \leq t \leq t_{0} \\ 1, & t_{0} \leq t \leq T .\end{cases}
$$

(3) If $\epsilon \geq \gamma_{1} e^{r T}$, the optimal reinsurance strategy is $q_{1}^{*}(t)=$ $1,0 \leq t \leq T$,

where $\epsilon$ is the unique positive root to (21). The corresponding optimal investment strategy is

$$
\pi_{1}^{*}(t)=\frac{\rho \beta}{\sigma}+\frac{\alpha-r}{\sigma^{2}} \frac{e^{r(t-T)}}{\gamma_{1}}, \quad 0 \leq t \leq T .
$$

\section{Optimal Strategy for the Reinsurer}

In this section, we discuss the optimal strategy for the reinsurer. Suppose that the utility function of the reinsurer is given by

$$
U_{2}(y)=\lambda_{1}-\frac{m}{\gamma_{2}} e^{-\gamma_{2} y}, \quad \gamma_{2}>0, m>0,
$$

where $\gamma_{2}$ represents the absolute risk aversion coefficient for the reinsurer.

For an admissible strategy $\left(q_{2}, \pi_{2}\right) \in \Pi_{2}$, the value function for the reinsurer is defined in the form

$$
H(t, y)=\sup _{\left(q_{2}, \pi_{2}\right)} E\left[U_{2}(Y(T)) \mid Y(t)=y\right],
$$


with $H(T, y)=U_{2}(y)$. The corresponding Hamilton-JacobiBellman (HJB) equation is

$$
\begin{aligned}
H_{t} & +[r y+\lambda \mu(1+\eta)] H_{y}+\sup _{\pi_{2}(t)}\left\{(\alpha-r) \pi_{2}(t) H_{y}\right. \\
& \left.+\frac{1}{2} \sigma^{2} \pi_{2}^{2}(t) H_{y y}\right\}+\sup _{q_{2}(t)}\left\{-\lambda \mu(1+\eta) q_{2}(t) H_{y}\right. \\
& \left.+\lambda E\left[H\left(t, y-\left(1-q_{2}(t)\right) P\right)-H(t, y)\right]\right\}=0
\end{aligned}
$$

with the boundary condition $H(T, y)=U_{2}(y)$, where $H_{t}, H_{y}$, and $H_{y y}$ denote partial derivatives of $H=H(t, y)$. The firstorder maximizing condition for the investment strategy $\pi_{2}(t)$ yields

$$
\pi_{2}^{*}(t)=-\frac{\alpha-r}{\sigma^{2}} \frac{H_{y}}{H_{y y}} .
$$

Substituting the optimal investment strategy $\pi_{2}^{*}(t)$ into the HJB equation (48), we derive that

$$
\begin{aligned}
& H_{t}+[r y+\lambda \mu(1+\eta)] H_{y}-\frac{(\alpha-r)^{2}}{2 \sigma^{2}} \frac{H_{y}^{2}}{H_{y y}} \\
& +\sup _{q_{2}(t)}\left\{-\lambda \mu(1+\eta) q_{2}(t) H_{y}\right. \\
& \left.+\lambda E\left[H\left(t, y-\left(1-q_{2}(t)\right) P\right)-H(t, y)\right]\right\}=0 .
\end{aligned}
$$

We formulate a solution for (50) in the form

$$
H(t, y)=\lambda_{1}-\frac{m}{\gamma_{2}} \exp \left[-\gamma_{2} y e^{r(T-t)}+k(T-t)\right]
$$

where $k(\cdot)$ is a suitable function such that formulation (51) is a solution of (50). The boundary condition $H(T, y)=U_{2}(y)$ implies $k(T)=0$. Using (51), we have

$$
\begin{aligned}
& H_{t}=\left(H(t, y)-\lambda_{1}\right)\left[\gamma_{2} y r e^{r(T-t)}-k^{\prime}(T-t)\right], \\
& H_{y}=\left(H(t, y)-\lambda_{1}\right)\left[-\gamma_{2} e^{r(T-t)}\right], \\
& H_{y y}=\left(H(t, y)-\lambda_{1}\right)\left[\gamma_{2}^{2} e^{2 r(T-t)}\right], \\
& E\left[H\left(t, y-\left(1-q_{2}(t)\right) P\right)-H(t, y)\right] \\
& \quad=\left(H(t, y)-\lambda_{1}\right)\left[M_{P}\left(\gamma_{2}\left(1-q_{2}(t)\right) e^{r(T-t)}\right)-1\right] .
\end{aligned}
$$

Substituting (52) into (50) gives rise to

$$
\begin{aligned}
H_{t}+ & {[r y+\lambda \mu(1+\eta)] H_{y}-\frac{(\alpha-r)^{2}}{2 \sigma^{2}} \frac{H_{y}^{2}}{H_{y y}} } \\
& +\sup _{q_{2}(t)}\left\{f\left(q_{2}(t)\right)\right\}=0,
\end{aligned}
$$

where

$$
\begin{aligned}
& f\left(q_{2}(t)\right)=\lambda\left(H(t, y)-\lambda_{1}\right)\left[\gamma_{2} \mu(1+\eta) q_{2}(t) e^{r(T-t)}\right. \\
& \left.\quad+M_{P}\left(\gamma_{2}\left(1-q_{2}(t)\right) e^{r(T-t)}\right)-1\right] .
\end{aligned}
$$

Differentiating (54) with respect to $q_{2}(t)$, we get

$$
\begin{gathered}
\frac{\partial f\left(q_{2}(t)\right)}{\partial q_{2}(t)}=\lambda \gamma_{2} e^{r(T-t)}\left(H(t, y)-\lambda_{1}\right) \\
\cdot\left[\mu(1+\eta)-E\left[P e^{\gamma_{2}\left(1-q_{2}(t)\right) P e^{r(T-t)}}\right]\right], \\
\frac{\partial^{2} f\left(q_{2}(t)\right)}{\partial q_{2}^{2}(t)}=\lambda \gamma_{2}^{2} e^{2 r(T-t)}\left(H(t, y)-\lambda_{1}\right) \\
\cdot E\left[P^{2} e^{\gamma_{2}\left(1-q_{2}(t)\right) P e^{r(T-t)}}\right]<0 .
\end{gathered}
$$

Thus, $f\left(q_{2}(t)\right)$ is concave and its maximizer $q_{2}(t)$ satisfies the equation

$$
\mu(1+\eta)=E\left[P e^{\gamma_{2}\left(1-q_{2}(t)\right) P e^{r(T-t)}}\right]=M_{P}^{\prime}(\epsilon),
$$

where $\epsilon:=\gamma_{2}\left(1-q_{2}(t)\right) e^{r(T-t)}$.

From Lemma 1, there exists a unique positive root $\epsilon$ for (56). Therefore, we have $q_{2}(t)=1-\left(\epsilon / \gamma_{2}\right) e^{-r(T-t)} \leq 1$. Recall that the optimal reinsurance proportion $q_{2}^{*}(t) \in[0,1]$. If $0 \leq$ $q_{2}(t) \leq 1$, the optimal reinsurance proportion $q_{2}^{*}(t)$ coincides with $q_{2}(t)$. If $q_{2}(t) \leq 0$, we let $q_{2}^{*}(t)=0$. If $q_{2}(t) \geq 1$, we set $q_{2}^{*}(t)=1$. The optimal reinsurance strategy for the reinsurer is discussed in the following three cases.

When $\epsilon \leq \gamma_{2}$, we have $1-\epsilon / \gamma_{2} \geq 0$ and $q_{2}(t)=$ $1-\left(\epsilon / \gamma_{2}\right) e^{-r(T-t)} \geq 0$ for any $t \in[0, T]$. Then the optimal reinsurance strategy is $q_{2}^{*}(t)=q_{2}(t), 0 \leq t \leq T$.

When $\gamma_{2} \leq \epsilon \leq \gamma_{2} e^{r T}$, we have $1-\epsilon / \gamma_{1}<0$. Let $t_{0}=$ $T+(1 / r) \ln \left(\gamma_{2} / \epsilon\right)$. Then $q_{2}(t)=1-\left(\epsilon / \gamma_{2}\right) e^{-r(T-t)}>0$ for $t \epsilon$ $\left[0, t_{0}\right]$, and $q_{2}(t) \leq 0$ for $t \in\left[t_{0}, T\right]$; the optimal reinsurance strategy is

$$
q_{2}^{*}(t)= \begin{cases}q_{2}(t), & 0 \leq t \leq t_{0}, \\ 0, & t_{0} \leq t \leq T .\end{cases}
$$

When $\epsilon \geq \gamma_{2} e^{r T}$, we have $q_{2}(t)=1-\left(\epsilon / \gamma_{2}\right) e^{-r(T-t)} \leq$ $1-\left(\epsilon / \gamma_{2}\right) e^{-r T}<0$ for any $t \in[0, T]$. Then the optimal reinsurance strategy is $q_{2}^{*}(t)=0,0 \leq t \leq T$. 
Substituting $q_{2}^{*}(t)$ into (54), the value of $f\left(q_{2}^{*}(t)\right)$ is expressed as

$$
f\left(q_{2}^{*}(t)\right)= \begin{cases}\lambda\left(H(t, y)-\lambda_{1}\right)\left[\mu(1+\eta)(\underline{\epsilon}-\epsilon)+M_{P}(\epsilon)-1\right], & q_{2}^{*}(t)=q_{2}(t), \\ \lambda\left(H(t, y)-\lambda_{1}\right)\left[M_{P}(\underline{\epsilon})-1\right], & q_{2}^{*}(t)=0,\end{cases}
$$

where $\underline{\epsilon}:=\gamma_{2} e^{r(T-t)}$.

Replacing the supremum in (53) by $f\left(q_{2}^{*}(t)\right)$ yields

$$
\begin{gathered}
H_{t}+[r y+\lambda \mu(1+\eta)] H_{y}-\frac{(\alpha-r)^{2}}{2 \sigma^{2}} \frac{H_{y}^{2}}{H_{y y}} \\
+\lambda\left(H(t, y)-\lambda_{1}\right) f_{2}\left(q_{2}^{*}(t)\right)=0,
\end{gathered}
$$

where

$$
\begin{aligned}
& f_{2}\left(q_{2}^{*}(t)\right) \\
& = \begin{cases}\mu(1+\eta)(\underline{\epsilon}-\epsilon)+M_{P}(\epsilon)-1, & q_{2}^{*}(t)=q_{2}(t), \\
M_{P}(\underline{\epsilon})-1, & q_{2}^{*}(t)=0 .\end{cases}
\end{aligned}
$$

Using the approach of Legendre transform, we derive that the dual equation of (59) is

$$
\begin{aligned}
g_{t}(t, z) & -r g(t, z) \\
+ & {\left[\frac{(\alpha-r)^{2}}{\sigma^{2}}-r-\lambda f_{2}\left(q_{2}^{*}(t)\right)\right] z g_{z}(t, z) } \\
+ & \frac{(\alpha-r)^{2} z^{2}}{2 \sigma^{2}} g_{z z}(t, z)-\lambda \mu(1+\eta)=0
\end{aligned}
$$

with boundary condition $g(T, z)=\left(U_{2}^{\prime}\right)^{-1}(z)$.

From the exponential utility given by (46) and the dual equation (61), we set that the boundary condition is

$$
g(T, z)=-\frac{1}{\gamma_{2}} \ln \frac{z}{m}
$$

Suppose that a solution to (61) has the form

$$
g(t, z)=-\frac{i(t)}{\gamma_{2}} \ln \frac{z}{m}+j(t)
$$

with $i(T)=1$ and $j(T)=0$. We have

$$
\begin{aligned}
& g_{t}(t, z)=-\frac{i^{\prime}(t)}{\gamma_{2}} \ln \frac{z}{m}+j^{\prime}(t), \\
& g_{z}(t, z)=-\frac{i(t)}{\gamma_{2} z}, \\
& g_{z z}(t, z)=\frac{i(t)}{\gamma_{2} z^{2}} .
\end{aligned}
$$

Substituting (64) into (61), we get

$$
\begin{aligned}
& -\frac{\ln (z / m)}{\gamma_{2}} i^{\prime}(t)+j^{\prime}(t)+\frac{r \ln (z / m)}{\gamma_{2}} i(t)-r j(t) \\
& -\left[\frac{(\alpha-r)^{2}}{2 \sigma^{2}}-r-\lambda f_{2}\left(q_{2}^{*}(t)\right)\right] \frac{i(t)}{\gamma_{2}} \\
& -(1+\eta) \lambda \mu=0 .
\end{aligned}
$$

We decompose (65) into two equations

$$
\begin{aligned}
& i^{\prime}(t)-r i(t)=0 \\
& j^{\prime}(t)-r j(t)-\left[\frac{(\alpha-r)^{2}}{2 \sigma^{2}}-r-\lambda f_{2}\left(q_{2}^{*}(t)\right)\right] \frac{i(t)}{\gamma_{2}} \\
& \quad-(1+\eta) \lambda \mu=0 .
\end{aligned}
$$

Taking the boundary conditions $i(T)=1$ and $j(T)=0$ into account, the solutions to (66) are

$$
\begin{aligned}
i(t)= & e^{r(t-T)}, \\
j(t) & \\
= & \frac{-(1+\eta) \lambda \mu\left(1-e^{r(t-T)}\right)}{r} \\
& +\left[\frac{(\alpha-r)^{2}}{2 \sigma^{2}}-r-\lambda f_{2}\left(q_{2}^{*}(t)\right)\right] \frac{(t-T) e^{r(t-T)}}{\gamma_{2}} .
\end{aligned}
$$

The above derivations lead to the following theorem.

Theorem 4. Assume that the reinsurer takes an exponential utility function in the form of (46). Let $t_{0}=T+(1 / r) \ln \left(\gamma_{2} / \epsilon\right)$, the optimal reinsurance-investment strategies for the reinsurer are given as follows:

(1) If $\epsilon \leq \gamma_{2}$, the optimal reinsurance strategies is $q_{2}^{*}(t)=$ $1-\left(\epsilon / \gamma_{2}\right) e^{-r(T-t)}, 0 \leq t \leq T$.

(2) If $\gamma_{2} \leq \epsilon \leq \gamma_{2} e^{r T}$, the optimal reinsurance strategy is

$$
q_{2}^{*}(t)= \begin{cases}1-\frac{\epsilon}{\gamma_{2}} e^{-r(T-t)}, & 0 \leq t \leq t_{0} \\ 0, & t_{0} \leq t \leq T\end{cases}
$$

(3) If $\epsilon \geq \gamma_{2} e^{r T}$, the optimal reinsurance strategy is $q_{2}^{*}(t)=$ $0,0 \leq t \leq T$, 
where $\epsilon$ is the unique positive root to (56). The corresponding optimal investment strategy for the reinsurer is

$$
\pi_{2}^{*}(t)=\frac{\alpha-r}{\sigma^{2}} \frac{e^{r(t-T)}}{\gamma_{2}}, \quad 0 \leq t \leq T .
$$

\section{Comparative Analyses and Numerical Illustrations}

5.1. Comparative Analyses on $q_{1}^{*}(t)$ and $q_{2}^{*}(t)$. A fair reinsurance contract should consider the interests of both insurer and reinsurer, while they both prefer to choose a reinsurance proportion for their own utility maximization. Assume that the insurer and the reinsurer have complete information on the risk of insurance and investment. If the optimal retention level chosen by the insurer is larger than that of the reinsurer, the reinsurer will accept the strategy. But in the opposite case, the reinsurer may oppose the contract proposed by the insurer. When $\gamma_{1}=\gamma_{2}$, according to their wealth processes, the sum of two optimal reinsurance ratios chosen by insurer and the reinsurer equals 1 , which reflects the game between the insurer and the reinsurer.

We give specialized analyses on $q_{1}^{*}(t)=q_{2}^{*}(t), q_{1}^{*}(t)>$ $q_{2}^{*}(t)$, and $q_{1}^{*}(t)<q_{2}^{*}(t)$. When $q_{1}^{*}(t)=q_{2}^{*}(t)$, the insurer and reinsurer reach a consensus on the retention proportion and they both obtain the maximum value of terminal wealth; the insurance contract is established. When $q_{1}^{*}(t)>q_{2}^{*}(t)$, the insurer's retention proportion is larger than that of the reinsurer. The insurer prefers to purchase less reinsurance and undertake more risk himself, while the reinsurer is willing to accept a higher level of reinsurance. When $q_{1}^{*}(t)<q_{2}^{*}(t)$, the insurer's retention proportion is smaller than that of the reinsurer. The insurer would like to spread more risks to the reinsurer, which is unacceptable for the reinsurer. The insurer may need to find other reinsurers to undertake the rest $q_{2}^{*}(t)-q_{1}^{*}(t)$ proportion of reinsurance.

5.2. Sensitivity Analyses and Numerical Illustrations. In this section, we focus on the exponential distribution of the claim size. Since $E\left[P_{i}\right]=\mu$, the density function of the claim size $P_{i}$ is given by $d(p)=(1 / \mu) e^{-(1 / \mu) p}, p>0$. Thus, we derive that the positive solution to (21) and (56) is

$$
\epsilon=\frac{1}{\mu}\left(1-\sqrt{\frac{1}{1+\eta}}\right) .
$$

Furthermore, the explicit expressions of the reinsurance strategies for the insurer and the reinsurer are

$$
\begin{aligned}
& q_{1}(t)=\frac{e^{r(t-T)}}{\mu \gamma_{1}}\left(1-\sqrt{\frac{1}{1+\eta}}\right), \\
& q_{2}(t)=1-\frac{e^{r(t-T)}}{\mu \gamma_{2}}\left(1-\sqrt{\frac{1}{1+\eta}}\right) .
\end{aligned}
$$

According to the analyses of previous sections, denote the optimal reinsurance strategies $q_{1}^{*}(t)=\min \left\{q_{1}(t), 1\right\}$ and $q_{2}^{*}(t)=\max \left\{q_{2}(t), 0\right\}$.

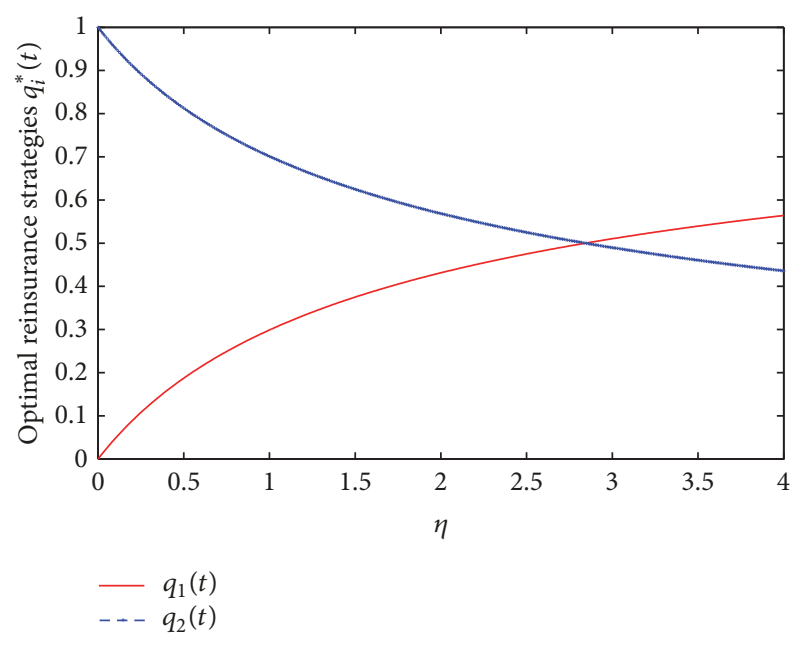

Figure 1: The effects of $\eta$ on $q_{i}^{*}(t)$.

In the following two subsections, we analyze the effects of market parameters on the optimal reinsurance-investment strategy and provide several numerical simulations to illustrate our results. Throughout the numerical analyses, unless otherwise stated, the parameters are given by $\beta=2, \mu=0.6$, $\eta=1, r=0.3, \alpha=0.5, \sigma=0.8, t=0$, and $T=7$.

5.2.1. Sensitivity Analysis on the Optimal Reinsurance Strategy. From (71), we derive that

$$
\begin{aligned}
& \frac{\partial q_{1}(t)}{\partial \eta}=\frac{e^{r(t-T)}}{2 \mu \gamma_{1}} \sqrt{\frac{1}{(1+\eta)^{3}}}>0, \\
& \frac{\partial q_{2}(t)}{\partial \eta}=-\frac{e^{r(t-T)}}{\mu \gamma_{2}} \sqrt{\frac{1}{(1+\eta)^{3}}}<0 .
\end{aligned}
$$

$q_{1}^{*}(t)$ is increasing with respect to the safety loading parameter $\eta$ while $q_{2}^{*}(t)$ is opposite. Figure 1 shows the effects of the safety loading parameter $\eta$ on the optimal reinsurance strategies. As $\eta$ becomes larger, the reinsurance premium will be more expensive. Thus the insurer prefers to retain a greater share of each claim and purchase less reinsurance. The reinsurer prefers to take more share of reinsurance to gain more profits.

From (71), we derive that

$$
\begin{aligned}
& \frac{\partial q_{1}(t)}{\partial \mu}=-\frac{e^{r(t-T)}}{\mu^{2} \gamma_{1}}\left(1-\sqrt{\frac{1}{1+\eta}}\right)<0, \\
& \frac{\partial q_{2}(t)}{\partial \mu}=\frac{e^{r(t-T)}}{\mu^{2} \gamma_{2}}\left(1-\sqrt{\frac{1}{1+\eta}}\right)>0,
\end{aligned}
$$

which implies that $q_{1}^{*}(t)$ decreases with $\mu$, as it is shown in Figure 2. Figure 2 also shows that $q_{2}^{*}(t)$ is an increasing function of $\mu$. This is that a greater $\mu$ means a larger claim size.

Figure 3 indicates the influence of risk-averse coefficients $\gamma_{i}$ on the reinsurance strategies. The insurer's risk aversion 


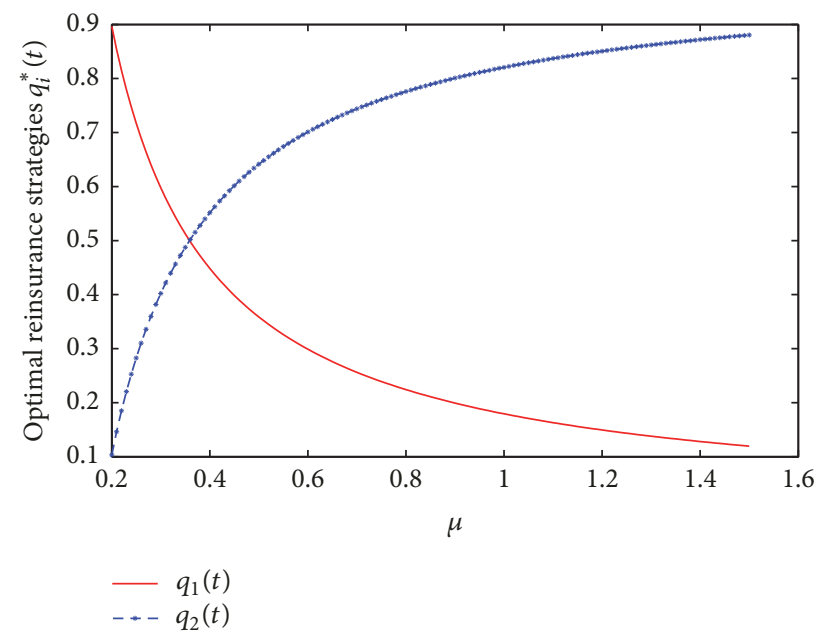

Figure 2: The effects of $\mu$ on $q_{i}^{*}(t)$.

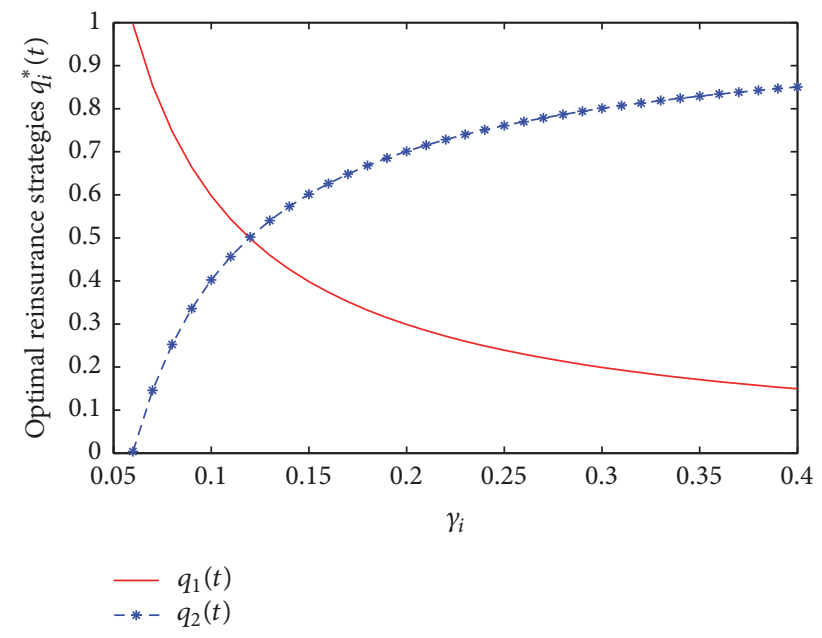

FIgURE 3: The effects of $\gamma_{i}$ on $q_{i}^{*}(t)$.

coefficient $\gamma_{1}$ for exponential utility exerts a negative effect on the retention $q_{1}^{*}(t)$ and the effect of $\gamma_{2}$ on $q_{2}^{*}(t)$ is positive. From (71), we derive that

$$
\begin{aligned}
& \frac{\partial q_{1}(t)}{\partial \gamma_{1}}=-\frac{e^{r(t-T)}}{\mu \gamma_{1}^{2}}\left(1-\sqrt{\frac{1}{1+\eta}}\right)<0, \\
& \frac{\partial q_{2}(t)}{\partial \gamma_{2}}=\frac{e^{r(t-T)}}{\mu \gamma_{2}^{2}}\left(1-\sqrt{\frac{1}{1+\eta}}\right)>0,
\end{aligned}
$$

which are consistent with the illustration in Figure 3. As the risk aversion coefficient becomes larger, the insurer would like to raise the reinsurance proportion to hedge risk, while the reinsurer becomes more conservative and will accept less reinsurance to reduce risk. This can be attributed to the fact that larger $\gamma_{i}$ means more risk-averse.

5.2.2. Sensitivity Analysis on the Optimal Investment Strategy. The effect of $\beta$ on the optimal investment strategy of the insurer with exponential utility is shown in Figures 4 and 5.

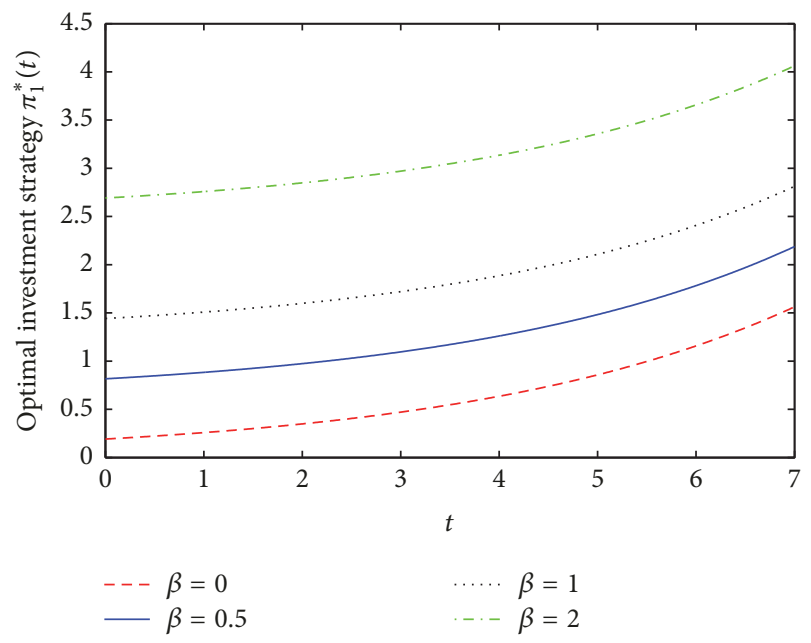

FIGURE 4: When $\rho=1$, the effects of $\beta$ on $\pi_{1}^{*}(t)$.

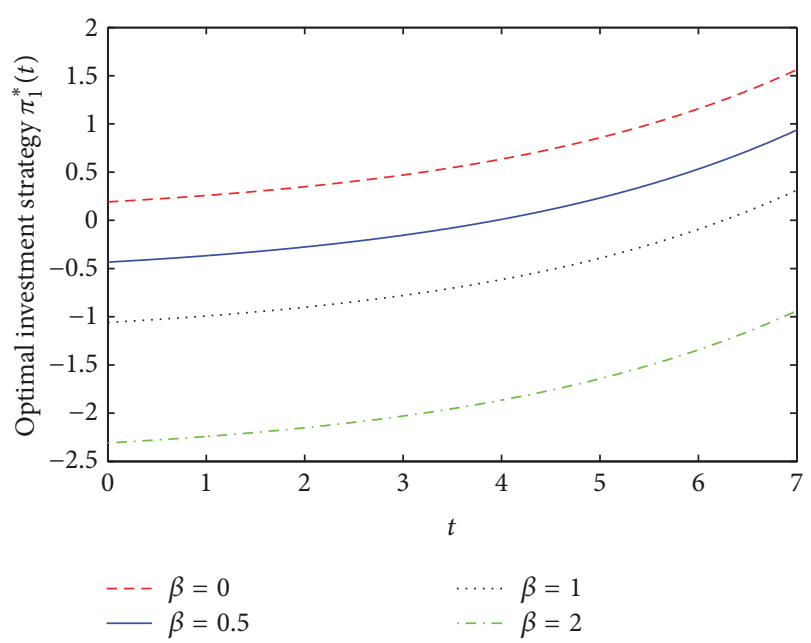

FIGURE 5: When $\rho=-1$, the effects of $\beta$ on $\pi_{1}^{*}(t)$.

From Figure 4 , we see that $\pi_{1}^{*}(t)$ increases with respect to the parameter $\beta$ in the case of $\rho=1$, which is due to the positive correlation between surplus process and stock price dynamics. The larger $\beta$, the more uncertainty on the surplus of the insurer. Thus the insurer would like to invest more in the financial market. If $\rho<0$, the correlation between surplus process of the insurer and stock price is negative; $\pi_{1}^{*}(t)$ decreases with respect to $\beta$; see Figure 5 .

From (4), we know that the parameter $\sigma$ represents the volatility of risky asset. The larger $\sigma$ is, the less wealth will be put into the risky assets, as it is depicted in Figure 6.

From Figure 7, we find that both $\pi_{1}^{*}(t)$ and $\pi_{2}^{*}(t)$ decrease with respect to the parameter $\gamma_{i}, i=1,2$. As $\gamma_{i}$ becomes larger, the investor is more risk adverse; they will reduce the amount in the risky assets.

\section{Conclusion}

In this paper, we investigate two optimization problems which take both the insurer and reinsurer into account. The 


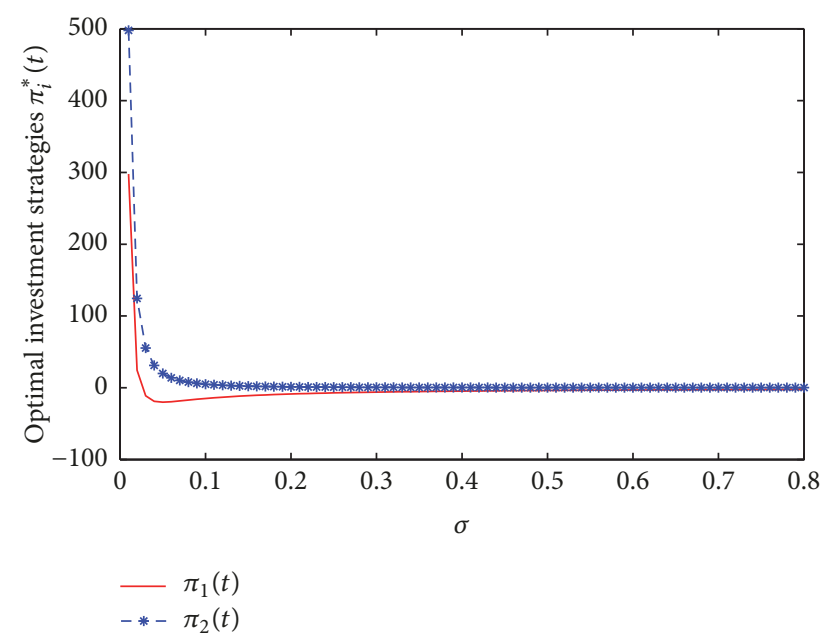

Figure 6: The effects of $\sigma$ on $\pi_{i}^{*}(t)$.

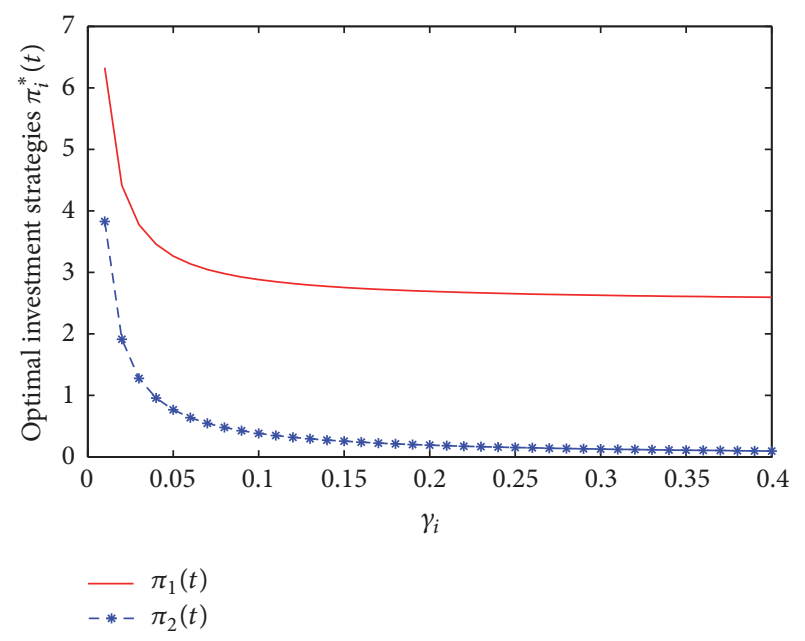

Figure 7: The effects of $\gamma_{i}$ on $\pi_{i}^{*}(t)$.

surplus process follows a jump-diffusion process and the insurer purchases proportional reinsurance from the reinsurer. Two wealth processes are described from the respective view of an insurer and a reinsurer, who are allowed to invest in a risky asset and a risk-free asset. Applying stochastic control theory, we derive the corresponding Hamilton-JacobiBellman equations and obtain the optimal reinsuranceinvestment strategies for exponential utility maximization. Comparing the optimal strategies designed by two sides, we observe that the insurer's reinsurance strategy is different from the reinsurer's strategy. The optimal reinsurance strategies are related to the safety loading of the reinsurer and the claim sizes distribution. If there exists a correlation between the risk model and the price of the risky asset, the optimal investment strategy is influenced by both financial market and insurance market. Otherwise, the investment strategy is only affected by the parameters in the financial market and the risk preference of the investor. When the insurer and the reinsurer have the same absolute reversion risk coefficient, the sum of two optimal proportion retentions equals 1, which implies the conflicting interests between the insurer and the reinsurer. Finally, we analyze the effects of several parameters on the optimal strategies and present several numerical simulations.

\section{Conflicts of Interest}

The authors declare that there are no conflicts of interest regarding the publication of this paper.

\section{References}

[1] H. Schmidli, "On minimizing the ruin probability by investment and reinsurance," The Annals of Applied Probability, vol. 12, no. 3, pp. 890-907, 2002.

[2] S. D. Promislow and V. R. Young, "Minimizing the probability of ruin when claims follow Brownian motion with drift," North American Actuarial Journal, vol. 9, no. 3, pp. 109-128, 2005.

[3] Z. Liang and J. Guo, "Optimal proportional reinsurance and ruin probability," Stochastic Models, vol. 23, no. 2, pp. 333-350, 2007.

[4] Y. Li and G. Liu, "Dynamic proportional reinsurance and approximations for ruin probabilities in the two-dimensional compound Poisson risk model," Discrete Dynamics in Nature and Society, vol. 2012, Article ID 802518, 26 pages, 2012.

[5] S. Luo and M. Taksar, "On absolute ruin minimization under a diffusion approximation model," Insurance: Mathematics \& Economics, vol. 48, no. 1, pp. 123-133, 2011.

[6] S. Browne, "Optimal investment policies for a firm with a random risk process: exponential utility and minimizing the probability of ruin," Mathematics of Operations Research, vol. 20, no. 4, pp. 937-958, 1995.

[7] H. Yang and L. Zhang, "Optimal investment for insurer with jump-diffusion risk process," Insurance: Mathematics \& Economics, vol. 37, no. 3, pp. 615-634, 2005.

[8] L. Bai and J. Guo, "Optimal proportional reinsurance and investment with multiple risky assets and no-shorting constraint," Insurance: Mathematics \& Economics, vol. 42, no. 3, pp. 968-975, 2008.

[9] Y. Cao and N. Wan, "Optimal proportional reinsurance and investment based on Hamilton-Jacobi-Bellman equation," Insurance: Mathematics \& Economics, vol. 45, no. 2, pp. 157-162, 2009.

[10] N. Bauerle, "Benchmark and mean-variance problems for insurers," Mathematical Methods of Operations Research, vol. 62, no. 1, pp. 159-165, 2005.

[11] X. Meng, X. Rong, L. Zhang, and Z. Du, "Worst-case investment and reinsurance optimization for an insurer under model uncertainty," Discrete Dynamics in Nature and Society, vol. 2016, Article ID 9693419, 8 pages, 2016.

[12] P. Chen and S. C. Yam, "Optimal proportional reinsurance and investment with regime-switching for mean-variance insurers," Insurance: Mathematics \& Economics, vol. 53, no. 3, pp. 871-883, 2013.

[13] H. Zhao, Y. Shen, and Y. Zeng, "Time-consistent investmentreinsurance strategy for mean-variance insurers with a defaultable security," Journal of Mathematical Analysis and Applications, vol. 437, no. 2, pp. 1036-1057, 2016.

[14] J. Cai and K. S. Tan, "Optimal retention for a stop-loss reinsurance under the VaR and CTE risk measures," The Journal of the International Actuarial Association, vol. 37, no. 1, pp. 93$112,2007$. 
[15] J. Cai, K. S. Tan, C. Weng, and Y. Zhang, "Optimal reinsurance under VaR and CTE risk measures," Insurance: Mathematics \& Economics, vol. 43, no. 1, pp. 185-196, 2008.

[16] A. Balbs, B. Balbs, and A. Heras, "Optimal reinsurance with general risk measures," Insurance: Mathematics \& Economics, vol. 44, no. 3, pp. 374-384, 2009.

[17] K. S. Tan, C. Weng, and Y. Zhang, "VaR and CTE criteria for optimal quota-share and stop-loss reinsurance," North American Actuarial Journal, vol. 13, no. 4, pp. 459-482, 2009.

[18] Y. Chi and K. S. Tan, "Optimal reinsurance under VaR and CVaR Risk measures: a simplified approach," ASTIN Bulletin, vol. 41, no. 2, pp. 487-509, 2011.

[19] K. Borch, “The optimal reinsurance treaty," ASTIN Bulletin, vol. 5, no. 2, pp. 293-297, 1969.

[20] D. Li, X. Rong, and H. Zhao, "Optimal reinsurance-investment problem for maximizing the product of the insurer's and the reinsurer's utilities under a CEV model," Journal of Computational and Applied Mathematics, vol. 255, pp. 671-683, 2014.

[21] D. Li, X. Rong, and H. Zhao, “Time-consistent reinsuranceinvestment strategy for an insurer and a reinsurer with meanvariance criterion under the CEV model," Journal of Computational and Applied Mathematics, vol. 283, pp. 142-162, 2015.

[22] J. Cai, Y. Fang, Z. Li, and G. E. Willmot, "Optimal Reciprocal Reinsurance Treaties Under the Joint Survival Probability and the Joint Profitable Probability," Journal of Risk and Insurance, vol. 80, no. 1, pp. 145-168, 2013.

[23] Y. Fang and Z. Qu, "Optimal combination of quota-share and stop-loss reinsurance treaties under the joint survival probability," IMA Journal of Management Mathematics, vol. 25, no. 1, pp. 89-103, 2014.

[24] Z. Liang, L. Bai, and J. Guo, "Optimal investment and proportional reinsurance with constrained control variables," Optimal Control Applications and Methods, vol. 32, no. 5, pp. 587-608, 2011.

[25] W. H. Soner, Controlled Markov Processes and Viscosity Solutions, Springer, Berlin, Germany, 1993.

[26] M. Jonsson, "Optimal investment problems and volatility homogenization approximations," 2002. 


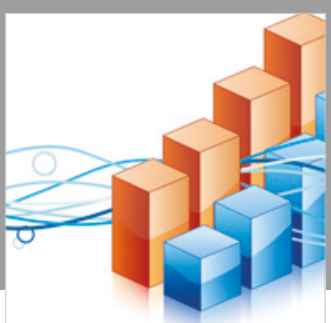

Advances in

Operations Research

\section{-n-m}
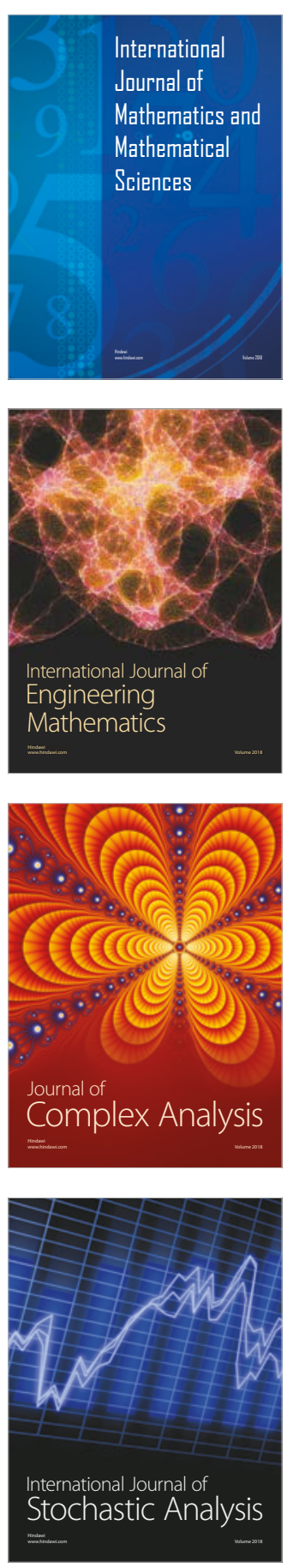
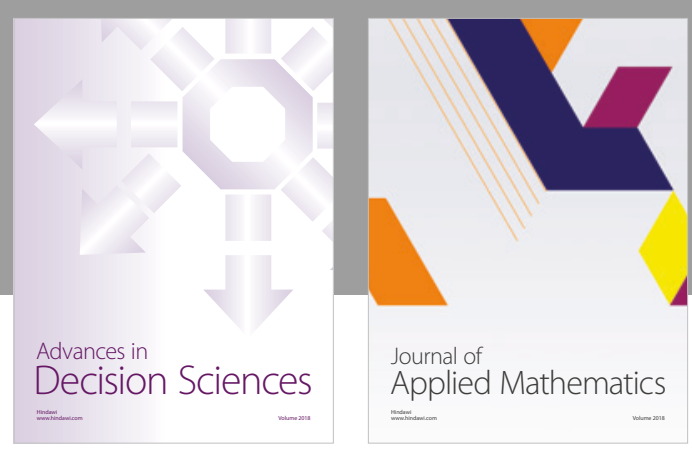

Journal of

Applied Mathematics
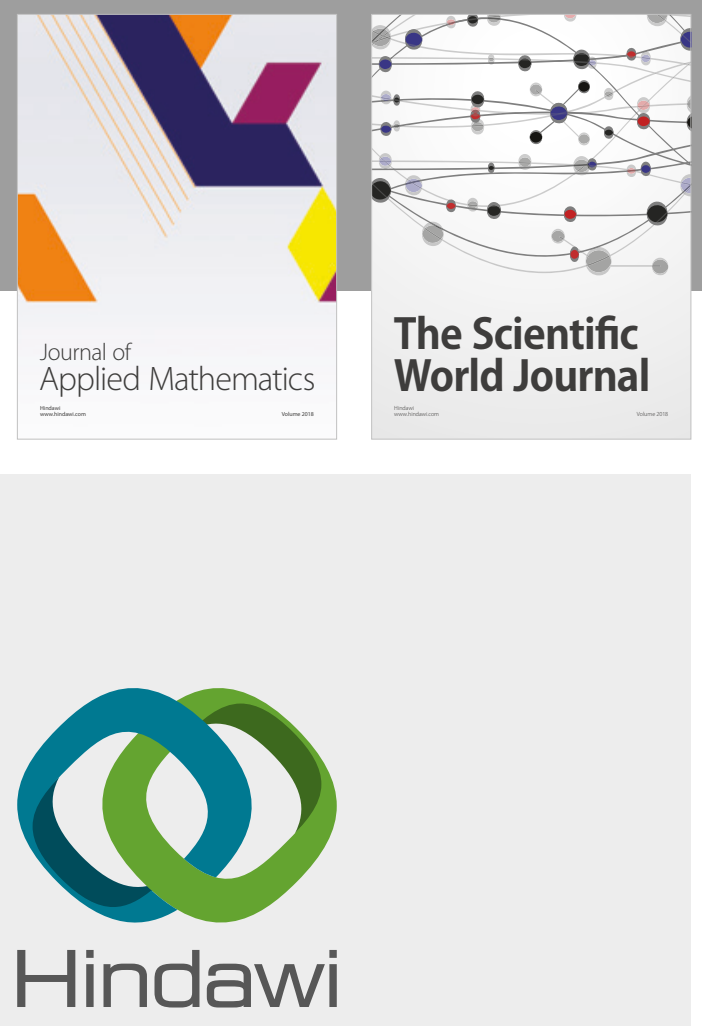

Submit your manuscripts at

www.hindawi.com

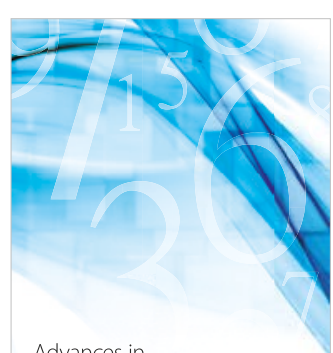

Advances in
Numerical Analysis
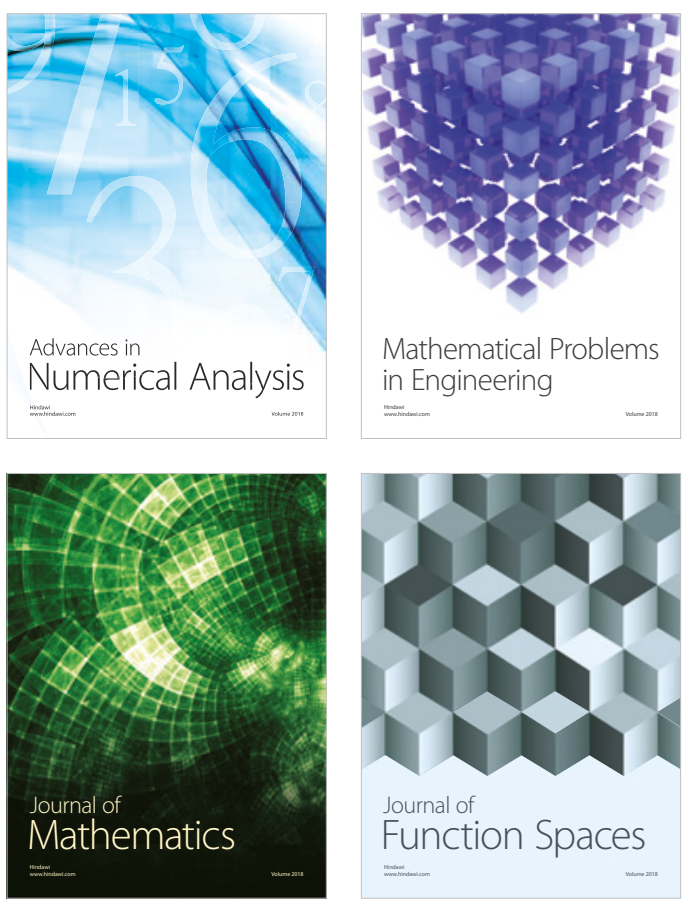

Mathematical Problems in Engineering

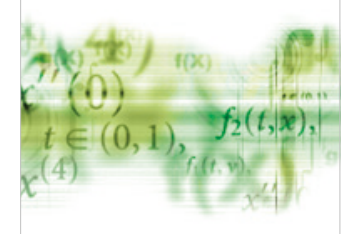

International Journal of

Differential Equations

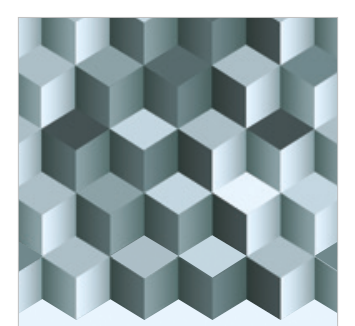

Journal of

Function Spaces

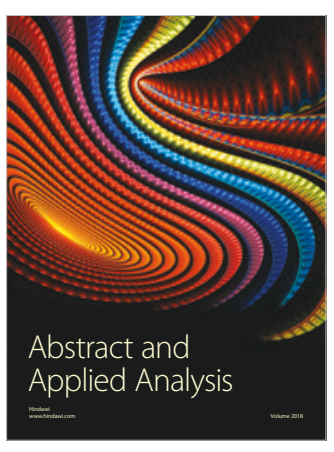

The Scientific

World Journal

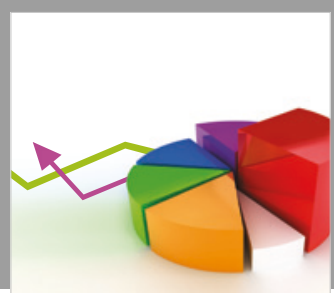

Journal of

Probability and Statistics
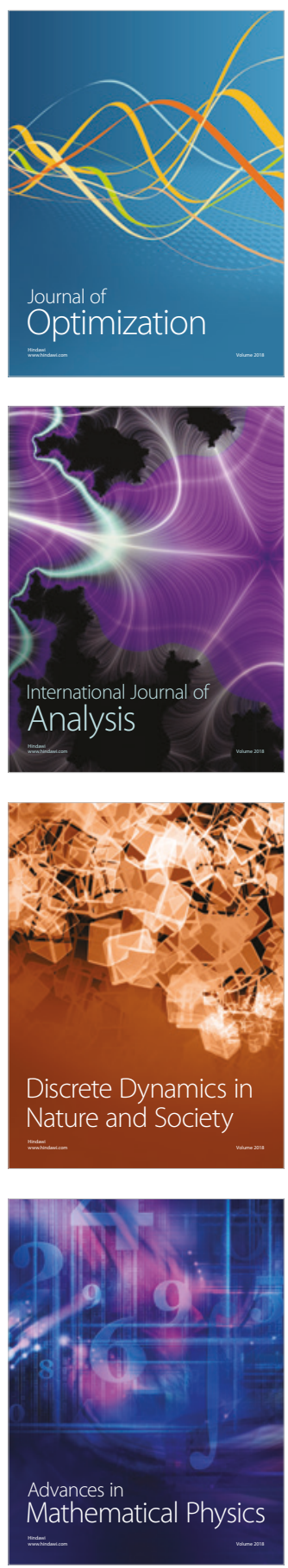